\title{
Computational fluid dynamic analysis on microvascular vein grafting: effect of mismatched conduit diameters
}

\begin{abstract}
An artery disease of upper extremity is uncommon happened. The most common cause of artery disease in upper extremity is atherosclerosis. In few patients with artery disease, surgical vein bypassing or vein interposition is frequently performed. However, one or more the internal diameters of applied vein graft are blocked or severely norrowed due to the mismatched diameter between existing artery and vein graft. The objective of this study is to investigate the blood flow influence on vein graft with mismatched diameter failure. The 3-D computational fluid dynamic method was employed to determine pulsatile flow velocity, pulsatile pressure gradient, and wall shear stress impact on the mismatched diameter of artery-vein graft model. We expect that pulsatile flow velocity, pulsatile pressure gradient, and wall shear stress impact on mismatched diameter of artery-vein graft model to behave non-hydraulically compared to an ideal matched model.
\end{abstract}

Keyword: Computational fluid dynamic; Numerical method; Vein graft survival; Digital artery disease; Upper extremity 